\title{
Endocytoscopy with NBI has the potential to correctly diagnose diminutive colorectal polyps that are difficult to diagnose using conventional NBI
}

\section{다)(1) $\odot(9$}

\author{
Authors \\ Shinichi Kataoka, Shin-ei Kudo, Masashi Misawa, Hiroki Nakamura, Kenichi Takeda, Naoya Toyoshima, Yuichi Mori, \\ Noriyuki Ogata, Toyoki Kudo, Tomokazu Hisayuki, Takemasa Hayashi, Kunihiko Wakamura, Toshiyuki Baba, Fumio \\ Ishida
}

\begin{abstract}
Institution
Digestive Disease Center, Showa University, Northern Yokohama Hospital, Yokohama, Japan
\end{abstract}

submitted 27.6.2019

accepted after revision 14.10.2019

\author{
Bibliography \\ DOI https://doi.org/10.1055/a-1068-9228 | \\ Endoscopy International Open 2020; 08: E360-E367 \\ (c) Georg Thieme Verlag KG Stuttgart · New York \\ eISSN 2196-9736
}

\section{Corresponding author}

Shin-ei Kudo, MD, PhD, Digestive Disease Center, Showa University, Northern Yokohama Hospital, 35-1 Chigasakichuo, Tsuzuki, Yokohama 224-8503, Japan

Fax: +81-45-949-7263

kudos@med.showa-u.ac.jp

\section{ABSTRACT}

Background and study aims Real-time diagnosis of colorectal polyps is needed to prevent unnecessary resection of benign polyps. The vessels in hyperplastic polyps sometimes mimic the characteristic meshed capillary network of neoplastic lesions on non-magnified narrow-band imaging (NBI). Endocytoscopy in conjunction with NBI (EC-
$\mathrm{NBI}$ ) enables more detailed vessel observation. The current study evaluated whether EC-NBI can accurately diagnose small colorectal lesions with visible vessels on non-magnified NBI.

Patients and methods This retrospective study was conducted from January to December 2016. During colonoscopy, lesion images were obtained using NBI and EC-NBI. On EC-NBI, lesions were classified as having "clear," "unclear," or "invisible" blood vessel margins. All specimens were resected and pathologically examined, and the association between vessel margin findings and pathological diagnosis was assessed. The lesion surface to vessel depth was measured in clear, unclear, and invisible lesions.

Results Among 114 adenomas, 108 were clear, while six were unclear. Among 36 hyperplastic polyps, eight were clear, while 28 were unclear. A micro-network (MN) pattern was seen in 106 of 114 adenomas, and four of 36 hyperplastic polyps. The sensitivity, specificity, correct diagnostic rate, and positive and negative predictive values of clear blood vessel margins or a MN pattern as an adenoma index were $98.2 \%, 69.4 \%, 91.3 \%, 91.1 \%$, and $92.6 \%$, respectively. EC-NBI correctly diagnosed $69.4 \%$ (25/36) of hyperplastic polyps. The lesion surface-blood vessel distance was greater in unclear versus clear lesions $(P<0.001)$, and invisible versus unclear lesions $(P<0.001)$.

Conclusions EC-NBI may effectively differentiate hyperplastic polyps with visible vessels from adenomas. Blood vessel depth affects visibility.

\section{Introduction}

Incidence of colorectal cancer (CRC) is currently increasing worldwide, and the disease has become one of the leading causes of death in western countries [1]. The most effective measure to prevent CRC is endoscopic resection of adenomatous polyps, which are the precursor lesions of CRC [2,3]. However, previous studies report that $28 \%$ to $36 \%$ of colorectal polyps are non-neoplasms that do not require resection, as they do not have malignant potential [4-7]. Thus, precise real- time diagnosis would enable the reduction of unnecessary endoscopic resection by leaving non-neoplastic lesions in situ; this would also reduce the cost related to unnecessary endoscopic resection and pathological diagnosis [8]. Therefore, the American Society for Gastrointestinal Endoscopy (ASGE) proposed the Prevention and Incorporation of Valuable Endoscopic Innovations (PIVI) statement [9].

Narrow-band imaging (NBI) is push-button image-enhanced endoscopy that may be effective in differentiating between neoplastic and non-neoplastic lesions [10-12]. Neoplastic le- 

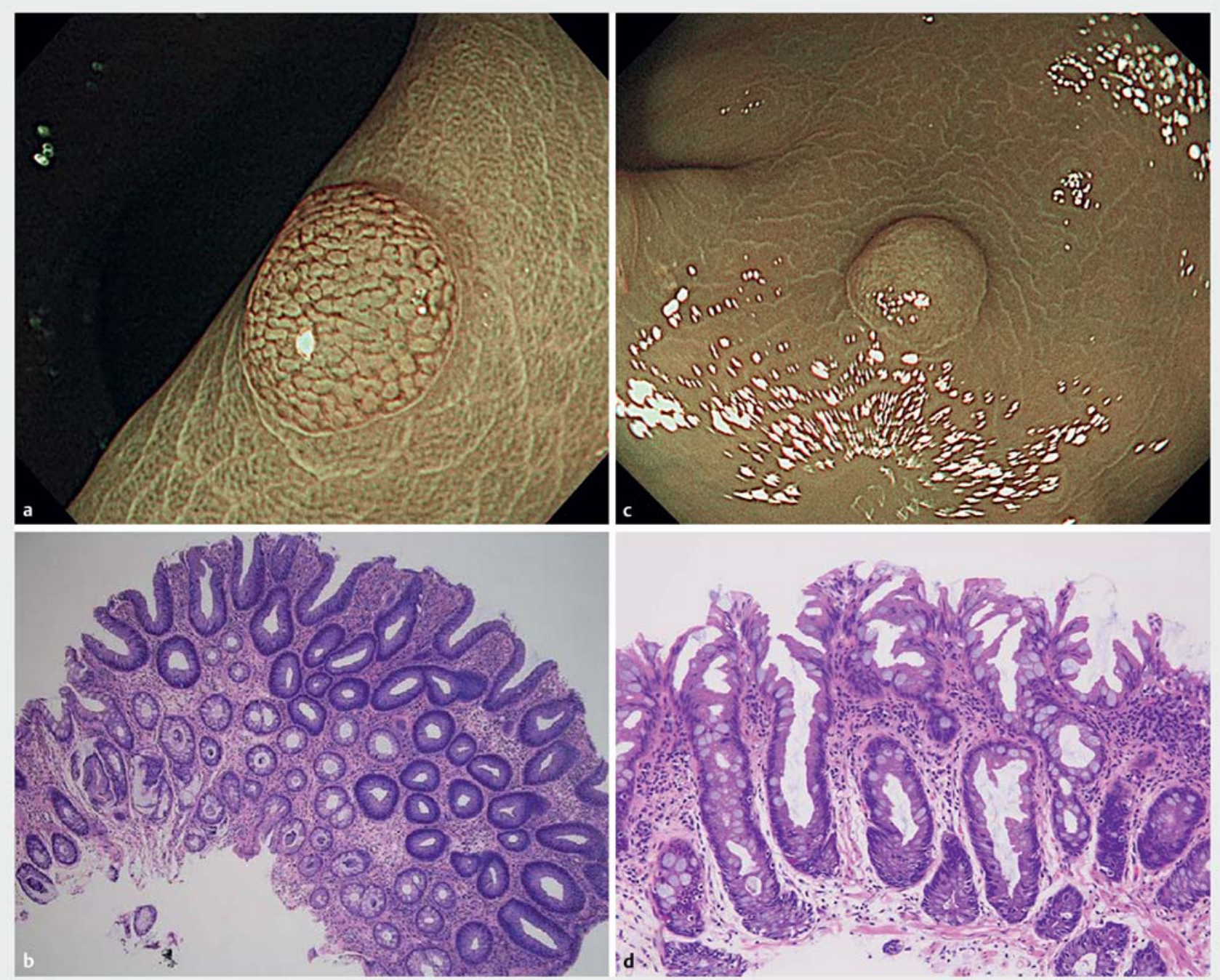

- Fig. 1 Images of a tubular adenoma and a hyperplastic polyp. a Tubular adenoma with a meshed capillary network visible on non-magnified narrow-band imaging (NBI). b Pathological section of the tubular adenoma shown in Fig. 1a. c Hyperplastic polyp with no visible meshed capillary network on non-magnified NBI. d Pathological section of the hyperplastic polyp shown in Fig. 1c.

sions usually have a meshed capillary network on magnified $\mathrm{NBI}$, and these meshed capillaries are specific for neoplasms [13] ( Fig. 1). However, recent large-scale prospective studies have not met the PIVI thresholds [4,5]. More than $10 \%$ of hyperplastic polyps reportedly contain blood vessels that look similar to the meshed capillary network observed in neoplasms [14]. Thus, these benign lesions might be misdiagnosed as malignant.

Endocytoscopy (EC) is an ultra-magnification endoscopy that enables the visualization of the glandular structure and cellular atypia in vivo, and is now commercially available in Japan and Western countries. We previously reported the effectiveness of EC in differentiating between neoplastic and nonneoplastic lesions [15-17]. Furthermore, EC in conjunction with NBI (EC-NBI) enables in vivo observation of blood vessels in more detail compared with images obtained using conventional magnification power without the use of any dye solution
[18]. However, it is still uncertain whether EC-NBI can be used to correctly diagnose the lesions that are difficult to diagnose using conventional NBI.

In the current study, we focused on non-neoplasms that had visible blood vessels on non-magnified NBI. We aimed to determine whether EC-NBI makes it possible to accurately diagnose these small vascular colorectal lesions that are difficult to diagnose using conventional NBI.

\section{Patients and methods}

This retrospective study was conducted at the Digestive Disease Center of Showa University, Northern Yokohama Hospital from January 2016 to December 2016. Inclusion criteria were: 1) lesions observed using both EC-NBI and non-magnified NBI; 2) endoscopically or surgically resected lesions; 3 ) lesions with a diameter $<5 \mathrm{~mm}$ (lesion diameter was measured using a treat- 
ment tool); 4) lesions with visible blood vessels on non-magnified NBI. Exclusion criteria were: 1) poor-quality imaging of the lesion (e.g. blurred image, bleeding, and/or dark image); 2) sessile serrated lesions; and 3) invasive cancer. The study protocol was approved by the Ethics Committee of Showa University Northern Yokohama Hospital (approval no. 17H005; approval date April 18, 2017). Before collection of the specimens, all participants gave written informed consent for the use of their endoscopic data for forthcoming retrospective analyses. The study was conducted in accordance with the Declaration of Helsinki.

\section{Colonoscopic procedure}

Before examination, patients underwent bowel preparation with 2 to $3 \mathrm{~L}$ of polyethylene glycol solution. Diazepam or midazolam and butyl-scopolamine or glucagon were administered intravenously for sedation and prevention of peristalsis. Colonoscopy was performed using a video processor system (EVIS LUCERA ELITE system; Olympus Corp., Tokyo, Japan) and a digital image filing system (Solemio Olympus Corp., Tokyo, Japan). An integrated-type endocytoscope with only one lens (CFY0058-I; Olympus Corp, Tokyo, Japan) with a 520-fold magnification with a focusing depth of $35 \mu \mathrm{m}$ was used. During the study period, the endocytoscope was a prototype model; however, the same endocytoscope (CF-H290ECl; Olympus Corp., Tokyo, Japan) was launched in 2018. This endocytoscope has a working length of $1330 \mathrm{~mm}$, an outside diameter of $12.8 \mathrm{~mm}$, and a 3.2-mm-diameter working channel. In the present study, the optical enhancement of EC-NBI was set at mode A8 and color mode 3.

When a lesion was found, the mucus adhering to its surface was washed off, and the lesion was observed under white light. The endoscopist then switched to NBI, and captured a nonmagnified image. To obtain an image using EC-NBI, the endoscopist first contacted the lesion with the lens of the endoscope, and then fully pulled down the zoom lever and pushed the capture button. Blood vessels could be evaluated without spraying when using NBI.

\section{Pathological evaluation}

All specimens were endoscopically resected and fixed in $10 \%$ buffered formalin solution immediately after retrieval. They were then stained with conventional hematoxylin and eosin. Pathological examination was performed based on the classification of the World Health Organization by a single pathologist who was blinded to the endoscopic data.

For lesions that were difficult for a single pathologist to diagnose, the pathology result was confirmed by a second expert pathologist.

\section{Evaluation of endocytoscopy in conjunction with NBI findings}

Microvessel findings on EC-NBI were assessed based on: (1) whether the margins of the blood vessels were clear or unclear and (2) presence or absence of a micro-network (MN) pattern. Lesions with blood vessels with clear margins were classified as "clear lesions," while those with blood vessels with unclear mar-

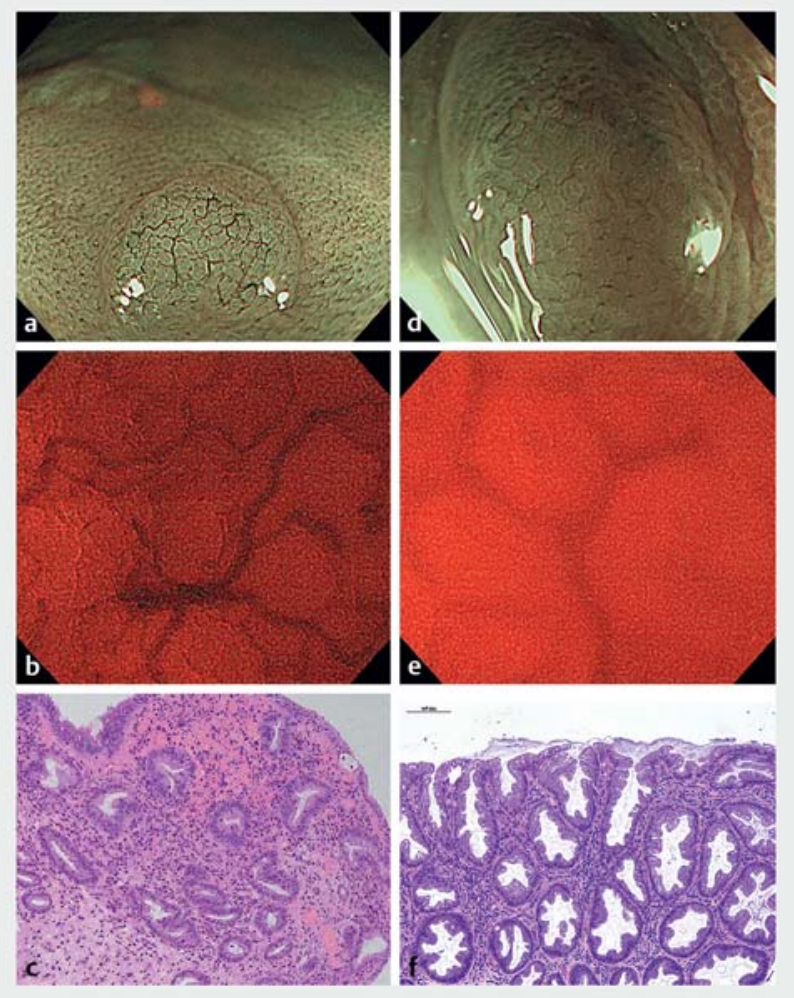

- Fig. 2 Images of a "clear lesion" and an "unclear lesion." a Hyperplastic polyp on non-magnified narrow-band imaging (NBI). b Endocytoscopic narrow-band imaging (EC-NBI) view of a "clear lesion" (the lesion shown in Fig. 2a). The margins of the blood vessels are clear. c Pathological section of the lesion shown in Fig. 2a. d Hyperplastic polyp on non-magnified NBI. e EC-NBI view of an "unclear lesion" (the lesion shown in Fig. 2d). The margins of the blood vessels are unclear. $\mathbf{f}$ Pathological section of the lesion shown in Fig. 2d.

gins were classified as "unclear lesions." A blood vessel that had discernible edges that could be followed was defined as having a clear margin, while a blood vessel that that had indiscernible edges that could not be followed was defined as having unclear margins ( $\triangleright$ Fig. 2 ). The MN pattern was defined as presence of microvessels in a characteristic meshed pattern in the intervening mucosa ( $\mathbf{F i g . 3}$ ). A meshed capillary pattern is shown using magnifying $\mathrm{NBI}$, whereas a MN pattern is shown using EC-NBI. The MN pattern is reportedly characteristic of adenomas $[11,19]$. We previously reported the endocytoscopic vascular (EC-V) pattern, which is classified as EC-V1 in hyperplastic polyps, EC-V2 in adenomas or intramucosal cancer, and EC-V3 in submucosal invasive cancer. Lesions with blood vessels with unclear margins are included in EC-V1, while lesions with blood vessels with clear margins or a MN pattern are included in ECV2. Thus, findings of clear or unclear blood vessel margins or a MN pattern complement the EC-V pattern findings. Microvessel findings on EC-NBI were assessed on still images by two endoscopists who were blinded to the pathological results. When the assessments were not consistent between the two endos- 


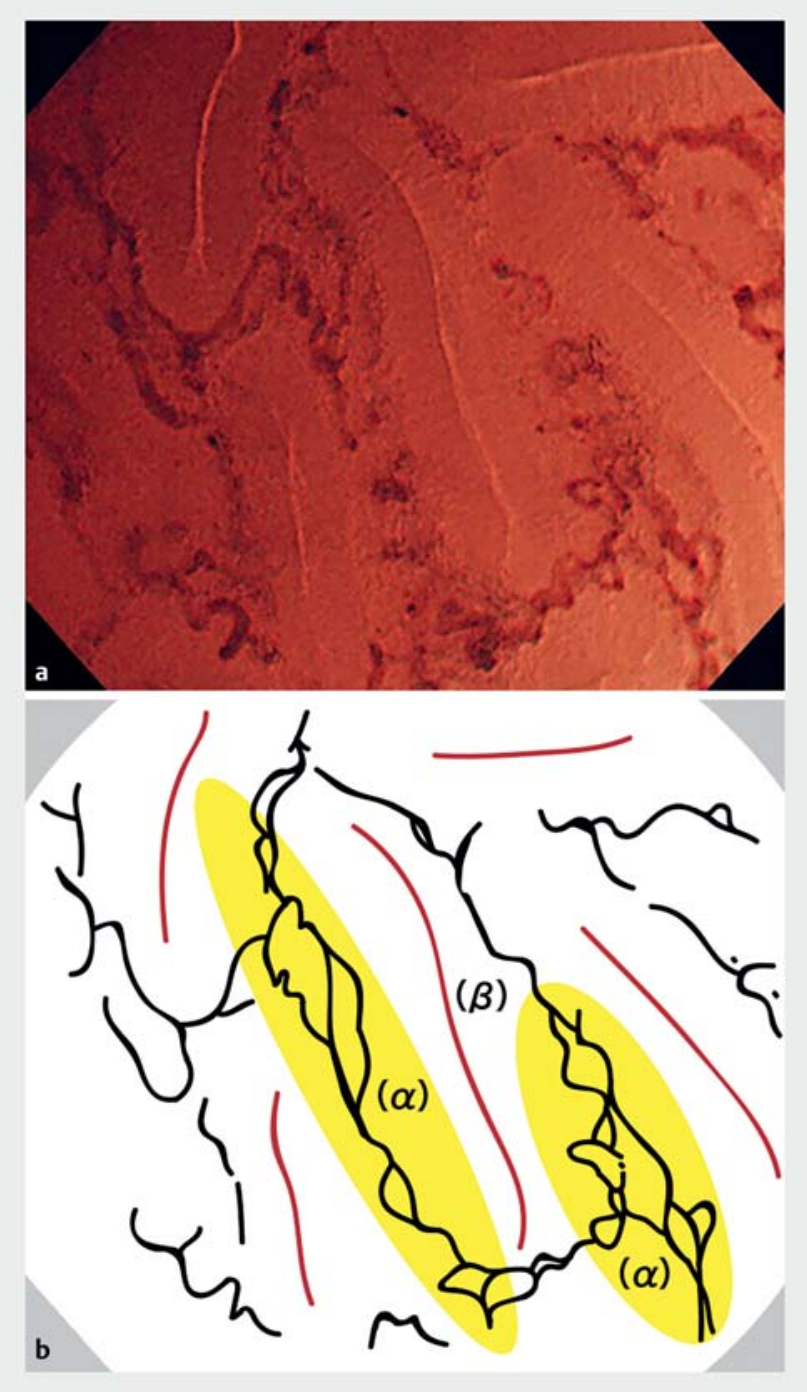

- Fig. 3 Typical micro-network (MN) pattern. a Endocytoscopic narrow-band imaging (EC-NBI) view of a typical adenoma. b Schematic representation of a MN pattern; a typical adenoma has fine and tortuous vessels in the intervening part (in the yellow line) around the crypt opening (?). Presence of these type of blood vessels was defined as the MN pattern. copists, the final consensus was achieved by discussion between the two endoscopists.

\section{Sub-study of the pathological factors associated with blood vessel findings}

We also assessed the correlation between the blood vessel margin findings observed using EC-NBI and pathological factors affecting these findings. In this assessment, we defined typical hyperplastic polyps that did not have visible blood vessels on $\mathrm{NBI}$ as "invisible lesions," in addition to clear lesions and unclear lesions ( $\mathbf{F i g . 4 )}$. We measured depth from the lesion surface to the blood vessels for each resected specimen using CD34 immunostaining. Measurements were made in 14 areas from four clear lesions, 14 areas from three unclear lesions, and 17 areas from three invisible lesions. The four clear lesions were all adenomas, while the three unclear lesions and three invisible lesions were all hyperplastic polyps. Areas that were not diagonally sliced were selected for measurement. We used cellsens software (Olympus Corp, Tokyo, Japan) for measurements.

\section{Sub-study for reproducibility}

We calculated interobserver agreement between experienced endoscopists regarding presence of a clear margin or a MN pattern as an adenoma index. An endoscopist who was not involved in the assessment evaluated 35 randomly selected EC images of the target lesions. The $\mathrm{K}$ statistic was used to calculate the interobserver agreement. A $\mathrm{k}$ value of 0.00 indicated poor agreement, $0.00-0.20$ indicated slight agreement, $0.21-$ 0.40 indicated fair agreement, $0.41-0.60$ indicated moderate agreement, $0.61-0.80$ indicated substantial agreement, and 0.80-1.00 indicated almost perfect agreement.

\section{Statistical analysis}

The BellCurve for Excel version 20.0 statistical software package (Social Survey Research Information Co., Ltd.) was used for data analysis. The Student's $t$-test was applied for the comparisons of continuous variables, and $P<0.05$ was considered significant.
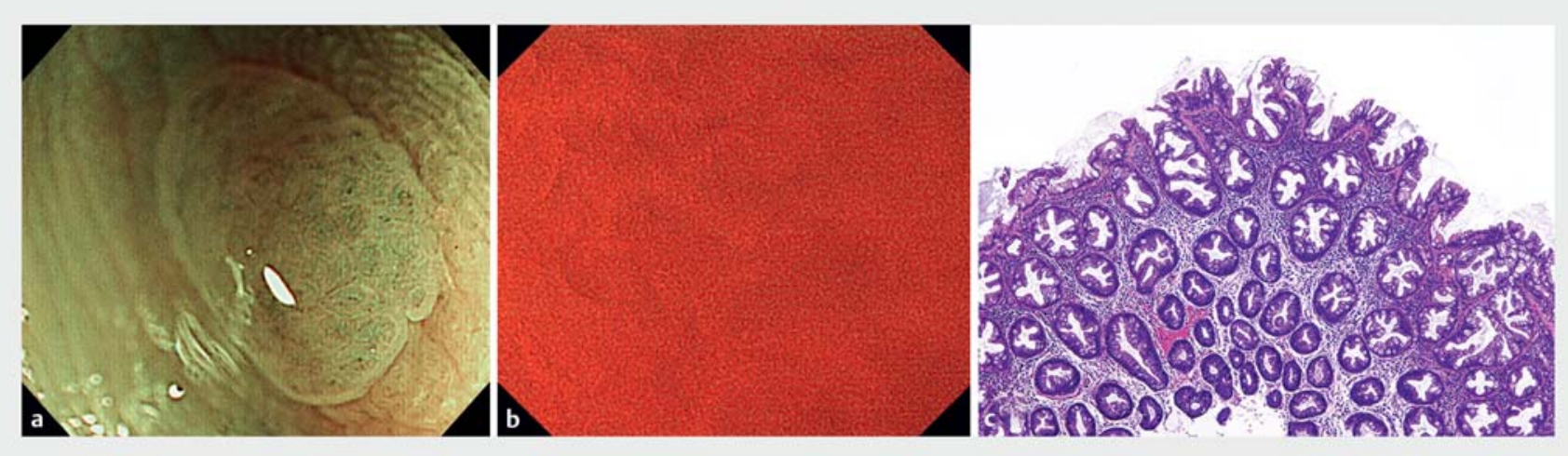

- Fig. 4 Images of an "invisible lesion." a Hyperplastic polyp defined as an "invisible lesion." b Endocytoscopic narrow-band imaging view of an "invisible lesion." Margins of the blood vessels are invisible. c Pathological section of the lesion shown in Fig. $\mathbf{4 a .}$ 


\section{Endocytoscopy was performed on 434 lesions from 255 patients \\ Excluded cases $(n=284)$ \\ - lesion diameter of more than $6 \mathrm{~mm}$ $(n=158)$ \\ - sessile serrated lesion $(n=4)$ \\ - poor quality images of the lesion $(n=69)$ \\ - lesions did not have visible blood vessels on non-magnified narrow-band imaging $(n=53)$ \\ Analysis of 150 lesions from 121 patients}

Fig. 5 Study flowchart.

\section{Results}

- Fig. 5 shows the flowchart for the analysis. A total of 434 lesions from 255 patients were resected after being observed with EC during the study period. Of these, 284 lesions were excluded for various reasons. Patient demographics and lesion characteristics are summarized in $>$ Table 1 .

- Table 2 shows the blood vessel margin findings in accordance with the pathological diagnoses. Among 114 tubular adenomas, 108 were clear lesions, while six were unclear lesions. Among 36 hyperplastic polyps, eight were clear lesions, while 28 were unclear lesions. The sensitivity, specificity, correct diagnostic rate, positive predictive value (PPV), and negative predictive value (NPV) were $94.7 \%, 77.8 \%, 90.7 \%, 93.1 \%$, and $82.4 \%$, respectively.

- Table 3 shows presence or absence of a MN pattern in accordance with the pathological diagnoses. A MN pattern was seen in 106 of 114 adenoma lesions, and in four of 36 hyperplastic polyps. The sensitivity, specificity, correct diagnostic rate, PPV, and NPV were $93.0 \%, 88.9 \%, 92.0 \%, 96.4 \%$, and $80.0 \%$, respectively.

- Table 4 shows diagnostic accuracy of using presence of a clear lesion or a MN pattern as an adenoma index. The sensitivity, specificity, correct diagnostic rate, PPV, and NPV were $98.2 \%, 69.4 \%, 91.3 \%, 91.1 \%$, and $92.6 \%$, respectively. All 36 hyperplastic polyps were lesions with visible blood vessels on non-magnified NBI. We were also able to correctly diagnose $69.4 \%(25 / 36)$ of these lesions by adding EC-NBI.

\section{Sub-study results}

Mean distances between the lesion surface and blood vessels in clear, unclear, and invisible lesions were $25.2 \mu \mathrm{m}, 56.1 \mu \mathrm{m}$, and $97.5 \mu \mathrm{m}$, respectively ( $>$ Fig. 6 ). This distance in unclear lesions was significantly longer than the distance in clear lesions $(P<$ 0.001). Similarly, this distance in invisible lesions was significantly longer than the distance in unclear lesions $(P<0.001)$. The $\mathrm{k}$ value for interobserver agreement between experienced endoscopists was 0.772 .
- Table 1 Patient and lesion characteristics.

\begin{tabular}{|l|l|l|}
\hline & \multicolumn{2}{|c|}{ Pathological diagnosis } \\
\hline & Adenoma & Hyperplastic polyp \\
\hline Age (years), mean \pm SD & $67.4 \pm 9.14$ & $64.9 \pm 11.0$ \\
\hline Number of patients & 90 & 31 \\
\hline - Males & 53 & 17 \\
\hline - Females & 37 & 14 \\
\hline Lesion size (mm), mean \pm SD & $3.20 \pm 0.63$ & $2.66 \pm 0.63$ \\
\hline Lesion location & & \\
\hline - Right side of the colon & 75 & 4 \\
\hline - Left side of the colon & 31 & 10 \\
\hline - Rectum & 8 & 22 \\
\hline SD, standard deviation & & \\
\hline
\end{tabular}

- Table 2 Blood vessel margin findings in pathologically diagnosed adenomas versus hyperplastic polyps.

\begin{tabular}{|l|c|c|c|}
\hline & Adenoma & Hyperplastic polyp & Total \\
\hline Clear margin & 108 & 8 & 116 \\
\hline Unclear margin & 6 & 28 & 34 \\
\hline Total & 114 & 36 & 150 \\
\hline
\end{tabular}

- Table 3 Presence or absence of micro-network (MN) patterns in pathologically diagnosed adenomas versus hyperplastic polyps.

\begin{tabular}{|c|c|c|c|}
\hline & Adenoma & Hyperplastic polyp & Total \\
\hline $\mathrm{MN}(+)$ & 106 & 4 & 110 \\
\hline $\mathrm{MN}(-)$ & 8 & 32 & 40 \\
\hline Total & 114 & 36 & 150 \\
\hline
\end{tabular}

MN, micronetwork

- Table 4 Diagnostic accuracy of using presence of a clear margin or micro-network (MN) pattern as an adenoma index.

\begin{tabular}{|l|c|c|c|}
\hline & Adenoma & Hyperplastic polyp & Total \\
\hline $\begin{array}{l}\text { Clear margins or } \\
\text { MN(+) }\end{array}$ & 112 & 11 & 123 \\
\hline $\begin{array}{l}\text { Unclear margins } \\
\text { and MN(-) }\end{array}$ & 2 & 25 & 27 \\
\hline Total & 114 & 36 & 150 \\
\hline MN, micronetwork & & & \\
\hline
\end{tabular}

\section{Discussion}

The current study newly revealed two clinical findings. First, EC$\mathrm{NBI}$ was able to effectively differentiate hyperplastic polyps with visible blood vessels from adenomas, while differentiation between these two lesions is difficult using conventional NBI 

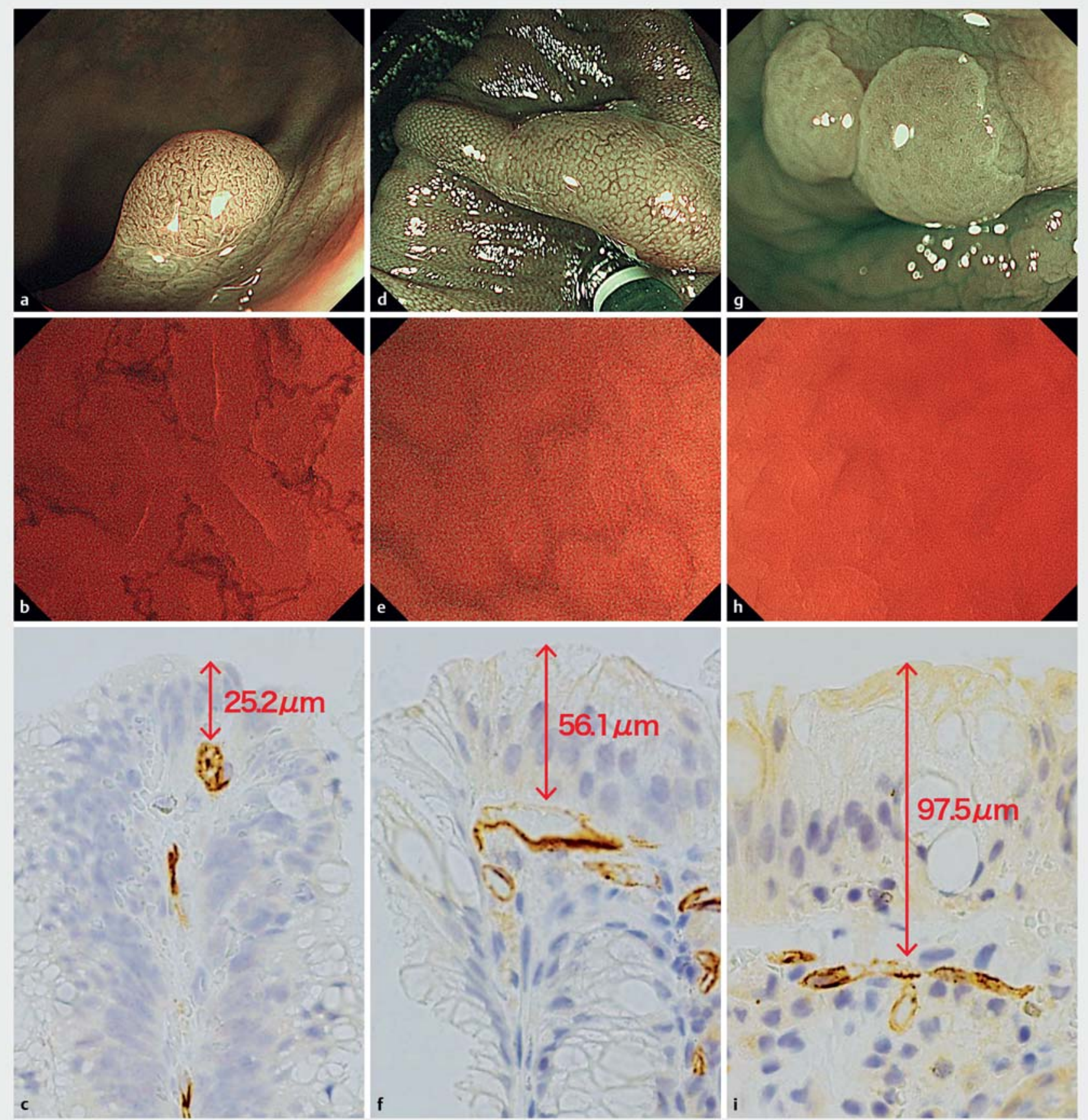

> Fig. 6 Mean distance between lesion surface and blood vessels. a Polyp defined as a "clear lesion" (tubular adenoma). b Endocytoscopic narrow-band imaging (EC-NBI) view of a "clear lesion." c Pathological section showing that the mean distance between the lesion surface and blood vessels of a "clear lesion" is $25.2 \mu \mathrm{m}$. $\mathbf{d}$ Polyp defined as an "unclear lesion" (hyperplastic polyp). e EC-NBI view of an "unclear lesion." $\mathbf{f}$ Pathological section showing that the mean distance between the lesion surface and blood vessels of an "unclear lesion" is $56.1 \mu \mathrm{m}$. $\mathbf{g}$ Polyp defined as an "invisible lesion" (hyperplastic polyp). h EC-NBI view of an "invisible lesion." i Pathological section showing that the mean distance between the lesion surface and the blood vessels of an "invisible lesion" is $97.5 \mu \mathrm{m}$.

observation. Second, visibility of blood vessels was affected by the depth of surface microvessels.

Our previous research found that EC-NBI has a significantly higher specificity than magnified NBI for predicting invasive cancer [18]. In the current study, we compared EC-NBI with non-magnified NBI. NBI can be used to visualize the surface mi- crovessels, and the presence of a surface meshed capillary network on colorectal polyps is a useful indicator of neoplasia [11]. This is because the blood vessels become thicker and more atypical as the grade of malignancy increases [20]. As adenomatous polyps have thick blood vessels and a surface meshed capillary network, these lesions appear brownish-colored on 
non-magnified NBI. In contrast, the blood vessels of hyperplastic polyps are thin, and so these lesions appear whitish-colored on NBI [21]. Based on these features, NBI can be used to differentiate polyps with visible blood vessels as neoplastic or nonneoplastic. Previous studies have reported contradictory findings regarding the real-time diagnosis of lesions using NBI. Sano et al. reported that the observation of surface meshed capillary vessels using magnifying $\mathrm{NBI}$ is a useful and simple method for differentiating non-neoplastic and neoplastic colorectal polyps [11]. Similarly, Kaltenbach et al. reported that optical diagnosis of diminutive colorectal polyps using NBI with a near focus view increases the confidence level of the optical diagnosis [12]. However, in contrast, Ladabaum et al. reported that only $25 \%$ of gastroenterologists were able to use realtime optical biopsy analysis of polyps using NBI to diagnose polyps with $\geq 90 \%$ accuracy [5]. Furthermore, Rees et al. stated that NBI-assisted optical diagnosis cannot currently be recommended for routine use outside of expert centers [4]. At least $10 \%$ of hyperplastic polyps reportedly have visible blood vessels [14]; these hyperplastic polyps with visible blood vessels are the likely cause of the misdiagnoses, and we consider that these types of lesions are the likely cause of diagnostic failure in previous studies. In the present study, EC-NBI was successfully used to diagnose these hyperplastic polyps with visible blood vessels, with a NPV of $92.6 \%$. To achieve the highest possible NPV, we adopted the combined diagnostic findings of both the blood vessel margins (clear or unclear) and presence or absence of a MN pattern. Although the current study was retrospective and had substantial bias, the NPV exceeded the threshold proposed by the ASGE. The PIVI statement proposed by the ASGE suggested that a modality with a NPV of over $90 \%$ for diagnosis of rectosigmoid diminutive polyps enables surgeons to leave hyperplastic polyps in situ without histopathological diagnosis [9]. Thus, it is necessary to correctly diagnose hyperplastic polyps with visible blood vessels as non-neoplastic rather than neoplastic. It is also important to avoid missing adenomas, which requires a high sensitivity of the diagnostic method. In the current study, although the specificity was not that high (69.4\%), the sensitivity was over $95 \%$. Furthermore, the diagnostic accuracy of EC-NBI as shown in Table 4 is relatively low compared with previous reports of the diagnostic accuracy of NBI with conventional optical zoom; this is because we focused on only the lesions that had visible blood vessels on non-magnified NBI. In addition, we have developed an artificial intelligence-assisted system for EC that is already available in Japan [22,23]. Such a computer-aided diagnostic system will be helpful for many endoscopists with insufficient EC experience.

We also found that depth of microvessels from the mucosal surface might influence visibility of microvessels. In the current study, visibility of surface blood vessels decreased as thickness of the epithelial layer increased, as the distance from the top layer to surface microvessels corresponds to the thickness of the epithelial layer. This indicates that hyperplastic polyps with visible blood vessels had a thinner epithelial layer compared with typical hyperplastic polyps with invisible blood vessels. EC-NBI could correctly distinguish a typical adenoma from a hy- perplastic polyp with visible blood vessels on non-magnified $\mathrm{NBI}$, as the focal depth of EC is $35 \mu \mathrm{m}$. Thus, blood vessels located deeper than the focal depth appear to have unclear margins during EC observations. In the sub-study, hyperplastic polyps with visible blood vessels on non-magnified NBI had a thicker epithelial layer compared with tubular adenomas $(56.1 \mu \mathrm{m}$ vs $25.2 \mu \mathrm{m}$ ). Thus, most of the hyperplastic polyps with visible blood vessels on non-magnified NBI had unclear blood vessel margins on EC-NBI, while adenomas had clear blood vessel margins on EC-NBI [24].

The current study has several limitations. First, it was a retrospective, single-center study. Thus, the required sample size was not calculated. We plan to increase the number of patients in a further prospective study. Second, there is a possibility that the distance between the lesion surface and blood vessels measured in the pathological specimen was shorter than the actual distance in the living body because of dehydration during specimen preparation. Third, this study did not include sessile serrated adenoma/polyps, as it is controversial whether this type of lesion is neoplastic $[25,26]$.

Fourth, only resected hyperplastic polyps were evaluated, without evaluating all of the hyperplastic polyps. Furthermore, size and location of the adenomas and hyperplastic polyps were not matched. Fifth, there was some polyp selection biases. Because the diminutive hyperplastic polyps included in the current study were often difficult to diagnose under endoscopy using EC, the polyps examined may differ from the polyps encountered in typical endoscopy.

\section{Conclusion}

In conclusion, EC-NBI might correctly diagnose diminutive colorectal polyps that are difficult to diagnose using non-magnified NBI. This might be because of the different epithelial thickness of these lesions, which was previously thought to be relatively uninformative data. EC-NBI has the potential to improve accuracy of diagnosis of small colorectal polyps, and enable the performance of optical biopsy.

\section{Acknowledgment}

The authors thank Kelly Zammit, BVSc, from Edanz Group (www.edanzediting.com/ac), for editing a draft of this manuscript.

\section{Competing interests}

Drs. S. Kudo and Misawa and Mori have received lecture fees from Olympus Corp.

References

[1] Arnold M, Sierra MS, Laversanne M et al. Global patterns and trends in colorectal cancer incidence and mortality. Gut 2017; 66: 683-691 
[2] Kaminski MF, Regula J, Kraszewska E et al. Quality indicators for colonoscopy and the risk of interval cancer. N Engl J Med 2010; 362: 1795-1803

[3] Zauber AG, Winawer S], O'Brien M] et al. Colonoscopic polypectomy and long-term prevention of colorectal-cancer deaths. N Engl J Med 2012; 366: 687-696

[4] Rees C], Rajasekhar PT, Wilson A et al. Narrow band imaging optical diagnosis of small colorectal polyps in routine clinical practice: the Detect Inspect Characterise Resect and Discard 2 (DISCARD 2) study. Gut 2017; 66: 887-895

[5] Ladabaum U, Fioritto A, Mitani A et al. Real-time optical biopsy of colon polyps with narrow band imaging in community practice does not yet meet key thresholds for clinical decisions. Gastroenterology 2013; 144: 81-91

[6] Rex DK, Overhiser AJ, Chen SC et al. Estimation of impact of American College of Radiology recommendations on CT colonography reporting for resection of high-risk adenoma findings. Am J Gastroenterol 2009; 104: 149-153

[7] Bond JH. Polyp guideline: diagnosis, treatment, and surveillance for patients with colorectal polyps. Practice Parameters Committee of the American College of Gastroenterology. Am J Gastroenterol 2000; 95: 3053-3063

[8] Ignjatovic A, East JE, Suzuki N et al. Optical diagnosis of small colorectal polyps at routine colonoscopy (Detect InSpect ChAracterise Resect and Discard; DISCARD trial): a prospective cohort study. Lancet Oncol 2009; 10: 1171-1178

[9] Rex DK, Kahi C, O’Brien M et al. The American Society for Gastrointestinal Endoscopy PIVI (Preservation and Incorporation of Valuable Endoscopic Innovations) on real-time endoscopic assessment of the histology of diminutive colorectal polyps. Gastrointest Endosc 2011; 73: 419-422

[10] Kudo S, Rubio CA, Teixeira CR et al. Pit pattern in colorectal neoplasia: endoscopic magnifying view. Endoscopy 2001; 33: 367-373

[11] Sano Y, Ikematsu H, Fu KI et al. Meshed capillary vessels by use of narrow-band imaging for differential diagnosis of small colorectal polyps. Gastrointest Endosc 2009; 69: 278-283

[12] Kaltenbach T, Rastogi A, Rouse RV et al. Real-time optical diagnosis for diminutive colorectal polyps using narrow-band imaging: the VALID randomised clinical trial. Gut 2015; 64: 1569-1577

[13] Sano Y, Muto M, Tajiri H et al. Optical/ digital chromoendoscopy during colonoscopy using narrow band imaging system. Dig Endosc 2005; 17: S43-\$48
[14] Uraoka T, Higashi R, Horii J et al. Prospective evaluation of endoscopic criteria characteristic of sessile serrated adenomas/polyps. J Gastroenterol 2015; 50: 555-563

[15] Kudo SE, Wakamura K, Ikehara $\mathrm{N}$ et al. Diagnosis of colorectal lesions with a novel endocytoscopic classification - a pilot study. Endoscopy 2011; 43: 869-875

[16] Mori Y, Kudo S, Ikehara N et al. Comprehensive diagnostic ability of endocytoscopy compared with biopsy for colorectal neoplasms: a prospective randomized noninferiority trial. Endoscopy 2013; 45: 98 105

[17] Kudo SE, Mori Y, Wakamura K et al. Endocytoscopy can provide additional diagnostic ability to magnifying chromoendoscopy for colorectal neoplasms. J Gastroenterol Hepatol 2014; 29: 83-90

[18] Kudo SE, Misawa M, Wada Y et al. Endocytoscopic microvasculature evaluation is a reliable new diagnostic method for colorectal lesions (with video). Gastrointest Endosc 2015; 82: 912-923

[19] Nakamura H, Kudo SE, Misawa M et al. Evaluation of microvascular findings of deeply invasive colorectal cancer by endocytoscopy with narrow-band imaging. Endosc Int Open 2016; 4: E1280-E1285

[20] Konerding MA, Fait E, Gaumann A. 3D microvascular architecture of pre-cancerous lesions and invasive carcinomas of the colon. $\mathrm{Br} J \mathrm{Can}$ cer 2001; 84: 1354-1362

[21] Wada Y, Kudo SE, Kashida $\mathrm{H}$ et al. Diagnosis of colorectal lesions with the magnifying narrow-band imaging system. Gastrointest Endosc 2009; 70: 522-531

[22] Mori Y, Kudo SE, Misawa M et al. Real-Time Use of artificial intelligence in identification of diminutive polyps during colonoscopy: a prospective study. Ann Intern Med 2018; 169: 357-366

[23] Misawa M, Kudo SE, Mori Y et al. Characterization of colorectal lesions using a computer-aided diagnostic system for narrow-band imaging endocytoscopy. Gastroenterology 2016; 150: 1531-1532 e3

[24] Hayashi T, Yatani R, Apostol J et al. Pathogenesis of hyperplastic polyps of the colon: a hypothesis based on ultrastructure and in vitro cell kinetics. Gastroenterology 1974; 66: 347-356

[25] Morson BC. Precancerous lesions of the colon and rectum. Classification and controversial issues. JAMA 1962; 179: 316-321

[26] Longacre TA, Fenoglio-Preiser CM. Mixed hyperplastic adenomatous polyps/serrated adenomas. A distinct form of coloreatal neoplasia. Am J Surg Pathol 1990; 14: 524-537 